\title{
Influence of a thin amorphous surface layer on de-channeling during aluminum implantation at different temperatures into $4 \mathrm{H}-\mathrm{SiC}$
}

\author{
M. K. Linnarsson ${ }^{1} \cdot$ A. Hallén ${ }^{2} \cdot$ L. Vines ${ }^{3}$
}

Received: 4 June 2019 / Accepted: 7 November 2019 / Published online: 19 November 2019

(c) The Author(s) 2019

\begin{abstract}
Ion implantation is an important technique in semiconductor processing and has become a key technology for $4 \mathrm{H}-\mathrm{SiC}$ devices. Today, aluminum ( $\mathrm{Al})$ implantations are routinely used for $\mathrm{p}$-type contacts, $\mathrm{p}^{+}$-emitters, terminations and many other applications. However, in all crystalline materials, quite a few ions find a path along a crystal channel, so-called channeling, and these ions travel deep into the crystal. This paper reports on the channeling phenomenon during $\mathrm{Al}$ implantation into $4 \mathrm{H}-\mathrm{SiC}$, and in particular, the influence of a thin native oxide will be discussed in detail. The effects of thermal lattice vibrations for implantations performed at elevated temperatures will also be elucidated. $100 \mathrm{keV} \mathrm{Al}$ ions have been implanted along the [000-1] direction employing samples with $4^{\circ}$ miscut. Before implantation, the samples have been aligned using the blocking pattern of backscattered protons. Secondary ion mass spectrometry has been used to record the Al depth distribution. To predict implantation profiles and improve understanding of the role of crystal structure, simulations were performed using the Monte-Carlo binary collision approximation code SIIMPL. Our results show that a thin surface layer of native oxide, less than $1 \mathrm{~nm}$, has a decisive role for de-channeling of aligned implantations. Further, as expected, for implantations at elevated temperatures, a larger degree of de-channeling from major axes is present due to increased thermal vibrations and the penetration depth of channeled aluminum ions is reduced. The values for the mean-square atomic displacements at elevated temperatures have been extracted from experimental depth profiles in combination with simulations.
\end{abstract}

\section{Introduction}

Ion implantation is an important technique in device manufacturing, in particular for silicon carbide (SiC), since diffusion of dopants is negligible at typical process temperatures $[1,2]$. For most of the technologically used doses, $\mathrm{SiC}$ will become damaged and the lattice structure will be hard to

M. K. Linnarsson

marga@kth.se

A. Hallén

ahallen@kth.se

L. Vines

lasse.vines@fys.uio.no

1 Materials and Nano-Physics, Department of Applied Physics, School of SCI, KTH Royal Institute of Technology, Electrum 229, 16440 Kista, Sweden

2 Department of Electronics, School of EECS, KTH Royal Institute of Technology, Electrum 229, 16440 Kista, Sweden

3 Physics Department/Center for Materials Science and Nanotechnology, University of Oslo, Blindern, P.O. Box 1048, 0316 Oslo, Norway restore if the implantation is performed at room temperature (RT). Therefore, ion implantations in $\mathrm{SiC}$ are usually performed at elevated temperature to preserve the crystallinity. In addition, high-temperature post-implantation annealing (above $1600^{\circ} \mathrm{C}$ ) is needed to recover the lattice and reach high dopant activation. Due to the negligible diffusivity of most dopants, these high-temperature activations do not significantly change the implantation profiles.

Typically, ion implantation is performed in some "random," non-channeling direction to minimize channeling effects. The standard miscut, $4^{\circ}$ off-axis from the [0001] toward the [11-20] direction, of $\mathrm{SiC}$ wafers is commonly used as a non-channeling direction for implantation into $4 \mathrm{H}-\mathrm{SiC}$. This results in a more or less Gaussian dopant depth distribution, where the depth is determined by the used ion energy and the target stopping power. However, a substantial amount of ions usually finds a crystal channel [3, 4], which results in a profile that extends considerably deeper than predicted and this may influence, for instance, the position of an electrical pn junction. On the other hand, if ion implantation is performed along, or close to, a major crystal direction, the channeled ions interact less with the target 
atoms, and therefore, the channeled ions may penetrate up to several times the projected range of the corresponding random implant in $4 \mathrm{H}-\mathrm{SiC}[3,5-8]$. In $4 \mathrm{H}-\mathrm{SiC}$ device design, several attempts have been made to take advantage of the channeling phenomena to create deep, box-shaped profiles $[9,10]$. However, most dopant implantations in SiC are performed at elevated temperature to suppress damage formation and hence thermal vibrations increase and less deep channeling profiles are expected at elevated temperatures. In addition to nuclear and electronic stopping, the extension of a channeled implantation profile is limited by the presence of a thin surface top layer consisting of adhesive contaminants and natural oxide. For example, in normal air ambient, a native oxide, $1 \mathrm{~nm}$ thick, is rapidly formed on $\mathrm{SiC}$ [11].

To support and explain experimental results, Lindhard developed in the 1960s a theoretical description of channeling where he introduced the concept of "critical angle for channeling" $[12,13]$. In this model, the probability for channeling is expressed as the maximum angle between the incoming beam and the axial row of atoms for which the ions still will be steered along the axis. The model is quite simple, based on atomic number, energy of impacting ions and atomic distances in the crystal, but the model has been very useful to predict experimental results for many semiconductors $[14,15]$. For more detail, three-dimensional MonteCarlo simulation codes have been developed for $\mathrm{SiC}$ using the binary collision approximation (MC-BCA) [16-20]. These codes originate from the algorithms described and implemented by Robinson et al. in the so-called MARLOWE code [21]. Unfortunately, the MC treatment is more time consuming compared to the straight forward concept of critical angle for channeling.

The increasing importance of $\mathrm{SiC}$ devices motivates us to take a closer look at channeling phenomena for Al implantations in $4 \mathrm{H}-\mathrm{SiC}$. In this study, two important aspects of channeling will be discussed: first, the effect from a thin amorphous, non-aligned, surface region and second, the influence from thermal vibrations during implantations at elevated temperatures. $100 \mathrm{keV} \mathrm{Al}{ }^{+}$ions have been employed for the implantation at temperatures between RT and $600{ }^{\circ} \mathrm{C}$. The ion beam has been aligned in parallel to the $4 \mathrm{H}-\mathrm{SiC}(0001)$ direction, and Monte-Carlo simulations using the binary collision approximation have been utilized to support the experimental results. 3D simulations have been employed to illustrate the expected distribution of channeled and de-channeled ions. It is shown that in addition to the used implantation temperature, the thin amorphous surface layer has a strong effect on the depth profiles.

\section{Experimental}

A $4 \mathrm{H}-\mathrm{SiC}$ wafer with a low doped, $5 \times 10^{15} \mathrm{~N} / \mathrm{cm}^{3}, 10 \mu \mathrm{m}$ epitaxial layer has been employed and samples of $5 \times 11$ $\mathrm{mm}^{2}$ size have been cut from the wafer. Before implantation, the sample was aligned and the ion beam struck the sample along the [000-1] direction. The error involved in the alignment of the crystal together with the beam divergence is $\leq 0.1^{\circ}$. For the sample alignment, $100 \mathrm{keV}$ protons were employed and a six-axis, high-precision goniometer was used. The blocking pattern for the backscattered protons was recorded with an area sensitive, 120-mm-diameter detector. After alignment, the source was reconfigured to produce $\mathrm{Al}$ ions.

Single-energy implants with $100 \mathrm{keV} \mathrm{Al}$ ions and doses between $2.5 \times 10^{12}$ and $2.5 \times 10^{14} \mathrm{~cm}^{-2}$ have been performed in different areas of the same sample. Typically, an area of $1.5 \times 1.5 \mathrm{~mm}^{2}$ was implanted and a low-dose rate was employed. Both room temperature (RT) and elevated temperatures have been used, i.e., the RT implantation was followed by (in situ) implantations at 600,400 and $200{ }^{\circ} \mathrm{C}$ in different areas of the same sample.

The $\mathrm{Al}$ depth distribution in the $4 \mathrm{H}-\mathrm{SiC}$ samples were determined by secondary ion mass spectrometry (SIMS) using a Cameca ims $7 \mathrm{f}$ instrument. A primary sputtering beam of $10 \mathrm{keV}^{32}\left(\mathrm{O}_{2}\right)^{+}$ions was applied and secondary ${ }^{27} \mathrm{Al}^{+}$ions were detected. The primary beam was rastered over an area of $200 \times 200 \mu^{2}$ and the ion signal was recorded from the central part of this area $(\sim 60 \mu \mathrm{m}$ in diameter) after which the crater depths were measured by a Dektak 8 stylus profilometer. A constant erosion rate was assumed for the conversion from sputter time versus depth. The exact implanted doses of the samples used in this study were determined by integrating the recorded SIMS profiles. For conversion of counts to concentrations in the recorded SIMS profiles, a $4 \mathrm{H}-\mathrm{SiC}$ reference sample implanted with $120 \mathrm{keV} \mathrm{Al}$ ions to a dose of $5 \times 10^{14} \mathrm{~cm}^{-2}$ has been employed.

\section{Simulation}

To improve the understanding of $\mathrm{Al}$ implantation profiles, experimental data have been combined with simulations using the Monte-Carlo binary collision approximation (MC-BCA) code SIIMPL [20]. The simulation code uses the universal potential of Ziegler, Biersack and Littmark (ZBL) [22], and scattering integrals are solved by the "Magic-Formula" [23]. In order to describe the lower electronic stopping in channels, the random electronic loss, $\mathrm{S}_{\mathrm{e}}$, is modified and given by, 
$\Delta E=S_{\mathrm{e}} A \exp \left(-s p / a_{\mathrm{u}}\right)$,

where $S_{\mathrm{e}}$ is expressed as $k E^{b}$ in the used range $\left(<E_{\mathrm{F}}\right.$, the Fermi energy) and the constants $k$ and $b$ are taken as 4.0 and 0.52 , respectively [24]. The electronic stopping is normalized by the constant $A, p$ is the impact parameter and $a_{\mathrm{u}}$ is the universal screening length corrected by a factor $s$. To estimate the number of displaced atoms, the model suggested by Kinching Pease [25] has been used. Displacement energies of 20 and $35 \mathrm{eV}$ have been utilized for $\mathrm{C}$ and $\mathrm{Si}$ atoms, respectively [26]. At elevated temperatures, increased dynamic annealing of damage has been accounted for [20]. Thermal vibrations have been included in the model according to the mean-square displacement of matrix atoms where values at RT are from reference 27 , while at elevated temperatures, mean-square displacements have been determined from fitting of implantations with different doses. The nonaligned, top layer is represented by an amorphous $\mathrm{SiC}$ layer in the simulations. In the SIIMPL code, for an amorphous target, the position of the next collision partner is randomly chosen in a cylinder in front of the ion and the type of ion is randomly chosen among the atoms defined in the unit cell, weighted by their relative abundance [20]. The density of amorphous $\mathrm{SiC}$ has been used in the simulations.

\section{Results and discussion}

Figure 1 shows a blocking pattern of backscattered protons from a $4 \mathrm{H}-\mathrm{SiC}$ sample. This kind of measurement has been utilized for sample alignment as the blocking pattern provides a map of planar and axial channels. The intensity is given by colors where dark blue stands for lower concentration of backscattering and represents high-symmetry axis

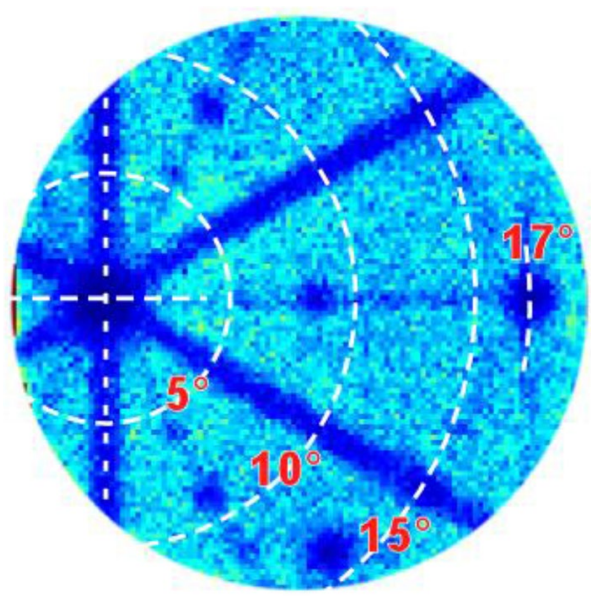

Fig. 1 Blocking pattern for $4 \mathrm{H}-\mathrm{SiC}$ probed with $100 \mathrm{keV}$ protons. Backscattered angles of $5^{\circ}, 10^{\circ}, 15^{\circ}$ and $17^{\circ}$ from $0^{\circ}$, the [0001] direction, are included in the polar map as white dotted lines and planes. The six-fold symmetry of $4 \mathrm{H}-\mathrm{SiC}$ is seen from the $\{11-20\}$ planes intersecting other $\{11-20\}$ planes along the [0001] direction. The off angles of $5^{\circ}, 10^{\circ}, 15^{\circ}$ and $17^{\circ}$ from the [0001] direction, used as the $0^{\circ}$ direction, in the polar map are indicated by white dotted lines. To the right, $17^{\circ}$ from [0001], in the $\{1-100\}$ plane, a large crystal channel, the $<11-23>$ direction, is clearly visible as a dark spot. For $\mathrm{Al}$ ions, a slightly larger critical angle for channeling is expected than for the protons used to collect the blocking pattern. This is due to a larger atomic number and lower velocity for $\mathrm{Al}$ ions compared to protons of the same energy [3, 5, 13]. As seen in Fig. 1, a large amount of possible channeling directions are available and after a few collisions, relatively independent of the impact angle, some ions will be guided into low index directions and planes of the crystal and these ions may be found deep into the crystal and also more laterally distributed than the original impact direction.

The 4H-SiC samples have been aligned and the $\mathrm{Al}$ implantations have been performed at RT along the [000-1] crystal direction. In Fig. 2, experimental Al depth profiles are shown for three implantations performed with doses $2.8 \times 10^{12}$ (low), $2.5 \times 10^{13}$ (medium) and $2.5 \times 10^{14}$ (high) $\mathrm{cm}^{-2}$, respectively. A logarithmic scale has been used for the concentration in Fig. 2. The lowest used dose is below the point where damage starts to accumulate in the channels and limit the ion paths. For this dose, the depth profile is box shaped with a small peak at $0.11 \mu \mathrm{m}$. As the dose increases,

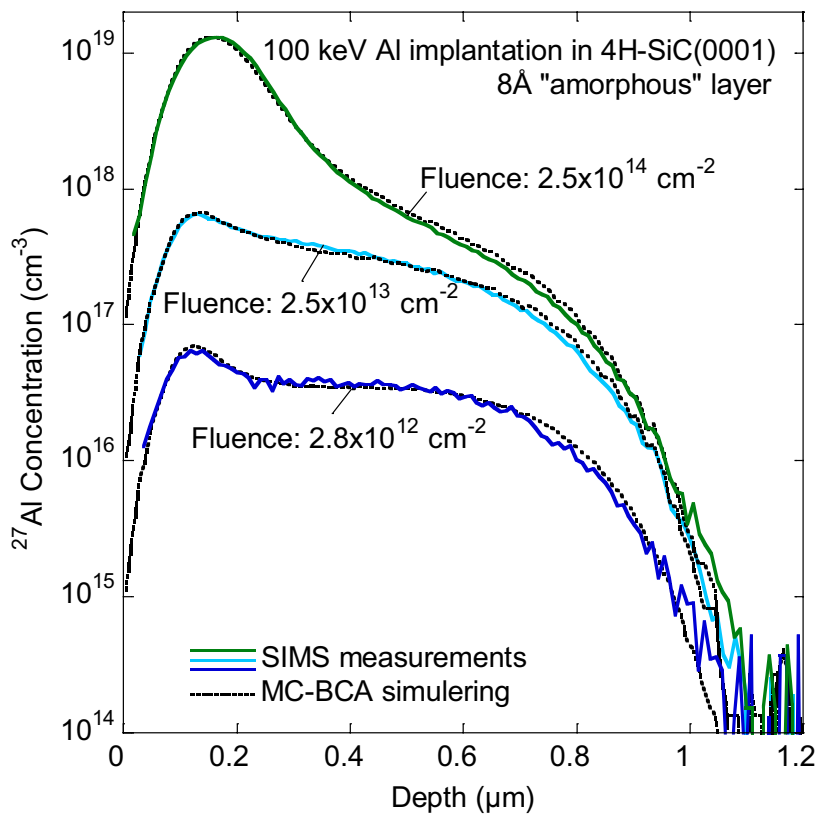

Fig. 2 SIMS measurements and MC-BCA simulation with the code SIIMPL of three $\mathrm{Al}$ implantations into $4 \mathrm{H}-\mathrm{SiC}$ parallel to the [0001] direction. $100 \mathrm{keV} \mathrm{Al}^{+}$ions and doses $2.8 \times 10^{12}, 2.5 \times 10^{13}$ and $2.5 \times 10^{14} \mathrm{~cm}^{-2}$ have been used. In the simulations, the native oxide is represented by an amorphous $8 \AA$ thick top layer 
the crystal channels will contain an increasing number of displaced $\mathrm{C}$ and $\mathrm{Si}$ atoms, and therefore, the probability for de-channeling of $\mathrm{Al}$ ions increases, and a small decrease in $\mathrm{Al}$ concentration with depth is observed for the medium dose. At the highest dose, a large number of interstitial atoms have blocked the channels and an Al peak starts to build-up similarly as in a random target. In addition to experimental SIMS results, MC-BCA simulations using the SIIMPL code have been included in Fig. 2. Fitting parameters used in these simulations will be discussed in the following text.

To further investigate the origin of the small peak observed at $0.11 \mu \mathrm{m}$ for the low- and medium-dose samples in Fig. 2, the effect from a thin, non-aligned top layer of various thicknesses on "de-channeling" is investigated by MCBCA simulations. Figure 3 illustrates this "de-channeling" effect of $\mathrm{Al}$ ions when passing through a thin amorphous $\mathrm{SiC}$ top layer, which is present on the single crystalline $4 \mathrm{H}-\mathrm{SiC}$ (0001) samples. The Al concentration is displayed versus depth for four different thicknesses, $0,4,8$ and $16 \AA$ of the amorphous surface layer, where the depth scale includes the surface layer thickness. In addition to simulations, experimental data has been included in Fig. 3. To avoid build-up of damage, the low-dose case (Fig. 2), $2.8 \times 10^{12} \mathrm{~cm}^{-2}$, has been used in the MC-BCA simulations. As the thickness of the amorphous layer increases, the scattering in the nonaligned surface layer increases and the peak around $0.11 \mu \mathrm{m}$ increases in height. This depth corresponds to the projected



Fig. 3 MC-BCA simulation of $\mathrm{Al}$ implantations into $4 \mathrm{H}-\mathrm{SiC}$, where the ions are entering along the [000-1] direction. An amorphous $\mathrm{SiC}$ surface top layer with a thickness of $0,4,8$ or $16 \AA$ has been assumed in the simulation using the SIIMPL code [20]. In addition, experimental data have been included range for implantations made in a non-channeling (random) direction according to the SRIM2013 code [22, 27]. In addition, the shape of the $\mathrm{Al}$ profile at depths between 0.3 and $1.1 \mu \mathrm{m}$ also changes and the concentration decreases faster with depth, as the amorphous layer thickness and the dechanneling of $\mathrm{Al}$ ions increase. Hence, in order to reproduce the experimental data between 0 and $0.2 \mu \mathrm{m}$, a scattering top layer has to be included in the simulations. If the native oxide of $4 \mathrm{H}-\mathrm{SiC}$ in Fig. 2 is represented by an $8 \AA$ thick amorphous layer, an excellent agreement between MC-BCA simulations and experimental data is obtained. This thickness corresponds well with the literature values of $9.3 \AA$, measured by angle-resolved $\mathrm{x}$-ray reflectivity, for a native oxide that consists mainly of silicon oxycarbide species after exposures of $\mathrm{SiC}$ to air [11]. The small discrepancy can be explained with a slightly lower density of the silicon oxycarbide layer compared to amorphous $\mathrm{SiC}$ utilized in the simulation.

For device applications, ion implantations are commonly performed at elevated temperatures to reduce the ioninduced damage and maintain the crystal structure. This will have a strong influence on channeled profiles, as previously discussed. In Fig. 4, implantations performed at RT as well as 200,400 and $600{ }^{\circ} \mathrm{C}$ are shown. The low-dose cases have been used to eliminate contribution from damage build-up and annealing. In the displayed depth profiles, a linear concentration scale has been used. As the temperature increases, the thermal vibrations increase and result in a larger probability for de-channeling and, therefore, less deep profiles. The effect from the upper most non-aligned layers is less visible as the temperature increases and it cannot be separated from the increasing contribution from de-channeling due to increasing lattice vibrations in the $600{ }^{\circ} \mathrm{C}$ depth profile. In the SIIMPL code, the thermal vibrations have been included at RT, as the mean-square displacement for carbon and silicon atoms, 0.051 and $0.057 \AA$, respectively, according to $\mathrm{x}$-ray diffraction measurements in $6 \mathrm{H}-\mathrm{SiC}$ [28]. The simplifying assumption taking the average of lattice vibrations over the crystal, independent of crystal directions, is used and any possible difference between $4 \mathrm{H}-\mathrm{SiC}$ and $6 \mathrm{H}-\mathrm{SiC}$ has been neglected. At elevated temperatures, the mean-square displacement values are determined from fitting of implantation depth distribution with different doses and temperatures. The values used are listed in Table 1. Note that a larger displacement is expected for carbon compared to silicon due to a lower mass. No detailed investigation has been made to separate the contribution from carbon and silicon vibrations and as a first approximation, the relative contribution from carbon and silicon has been scaled according to the relative mean-square displacement at RT for carbon and silicon. However, this ratio may decrease at elevated temperature as theoretical calculations suggest that the values of the meansquare displacement for carbon and silicon approach each 




Fig. $4 \mathrm{Al}$ concentration versus depth in four $4 \mathrm{H}-\mathrm{SiC}$ samples implanted at RT (a), $200{ }^{\circ} \mathrm{C}(\mathbf{b}), 400{ }^{\circ} \mathrm{C}(\mathbf{c})$ and $600{ }^{\circ} \mathrm{C}(\mathbf{d}) .100 \mathrm{keV}$ $\mathrm{Al}$ ions have been implanted along the [000-1] crystal direction. The SIMS data are drawn as solid lines while MC-BCA simulations are drawn as dotted lines

Table 1 Mean-square displacement for carbon and silicon atoms used in the SIIMPL code

\begin{tabular}{lll}
\hline Temperature & \multicolumn{2}{l}{ Mean-square displacement } \\
\cline { 2 - 3 } & Carbon atoms & Silicon atoms \\
\hline $\mathrm{RT}$ & $0.057 \AA$ & $0.051 \AA$ \\
$200{ }^{\circ} \mathrm{C}$ & 0.070 & 0.063 \\
$400{ }^{\circ} \mathrm{C}$ & 0.080 & 0.072 \\
$600{ }^{\circ} \mathrm{C}$ & 0.091 & 0.082 \\
\hline
\end{tabular}

other at high temperatures [28]. In this context, it should be pointed out that identical simulation curves can be obtained using the same mean-square displacement for both carbon and silicon. For example, at $400{ }^{\circ} \mathrm{C}$, using $0.074 \AA$ as the mean-square displacement for carbon and silicon gives the same simulation result for channeling along the [000-1] direction as the numbers 0.080 and $0.072 \AA$ for the meansquare displacement of carbon and silicon, respectively.

To better describe the native oxide at elevated temperatures, a $4 \AA$ thick, amorphous $\mathrm{SiC}$ top layer has been assumed in the MC-BCA simulations. Such a reduction in the layer thickness at 200,400 and $600{ }^{\circ} \mathrm{C}$ is motivated by desorption of surface contaminants during the treatment at $600{ }^{\circ} \mathrm{C}$ and it may also improve the lattice match of the native oxide. As seen from Fig. 4, the experimental data at different temperatures are perfectly reproduced in the simulations including the mentioned approximations of the meansquare displacement.

3D simulations have also been performed to trace the ion paths in detail and to include the contribution from dechanneling at elevated temperatures. Figure 5 displays 2D images sliced out from 3D simulations of $\mathrm{Al}$ implantations performed at temperatures between RT and $600{ }^{\circ} \mathrm{C}$ with doses according to the experimental data in Fig. 4. Figure 5 includes top views where the final position of all tracked ions is monitored as black dots and cross sections, i.e., thin slices of the $\{1-100\}$ plane, where the color gives the "intensity," or the probability, to find an ion at a particular position. The sample has been aligned and the incoming beam is along the [000-1] direction. In the simulations $5 \times 10^{6}$, ions have been tracked and a small impact area of $1 \mathrm{~nm}^{2}$, in which each ion position is randomly selected, has been used in the simulations. Such a small area is used to highlight preferential crystal directions and degree of de-channeling and to avoid overlapping and averaging of the output. Here it should be pointed out that if implantation is made with such a low dose in a device, as in Fig. 4, less than one ion will on average hit this small area and the simulation displays probability to find Al ions in a particular position. The intensity is displayed in colors utilizing a linear scale, where the highest probability is given in red and blue represents the lowest intensity. Different intensity scales have been used at different temperatures to easily find the depth for the maximum probability. Starting at the surface with an impact area of $1 \mathrm{~nm}^{2}$, at the depth of $0.11 \mu \mathrm{m}$ (the projected range in a random target), the lateral spread of ions is much larger and the probability to find an ion $0.1 \mu \mathrm{m}$ laterally from the original direction is high. For all temperatures up to and around the projected range, the distributions are similar. Below the random projected range, most of the ions follow the [000-1] channel but there is a blue, cone-shaped envelope of de-channeled ions. At RT, quite a few ions reach a depth of $1.1 \mu \mathrm{m}$ and the highest probability to find an ion in the original channel direction 
Top-view, position of all traced ions

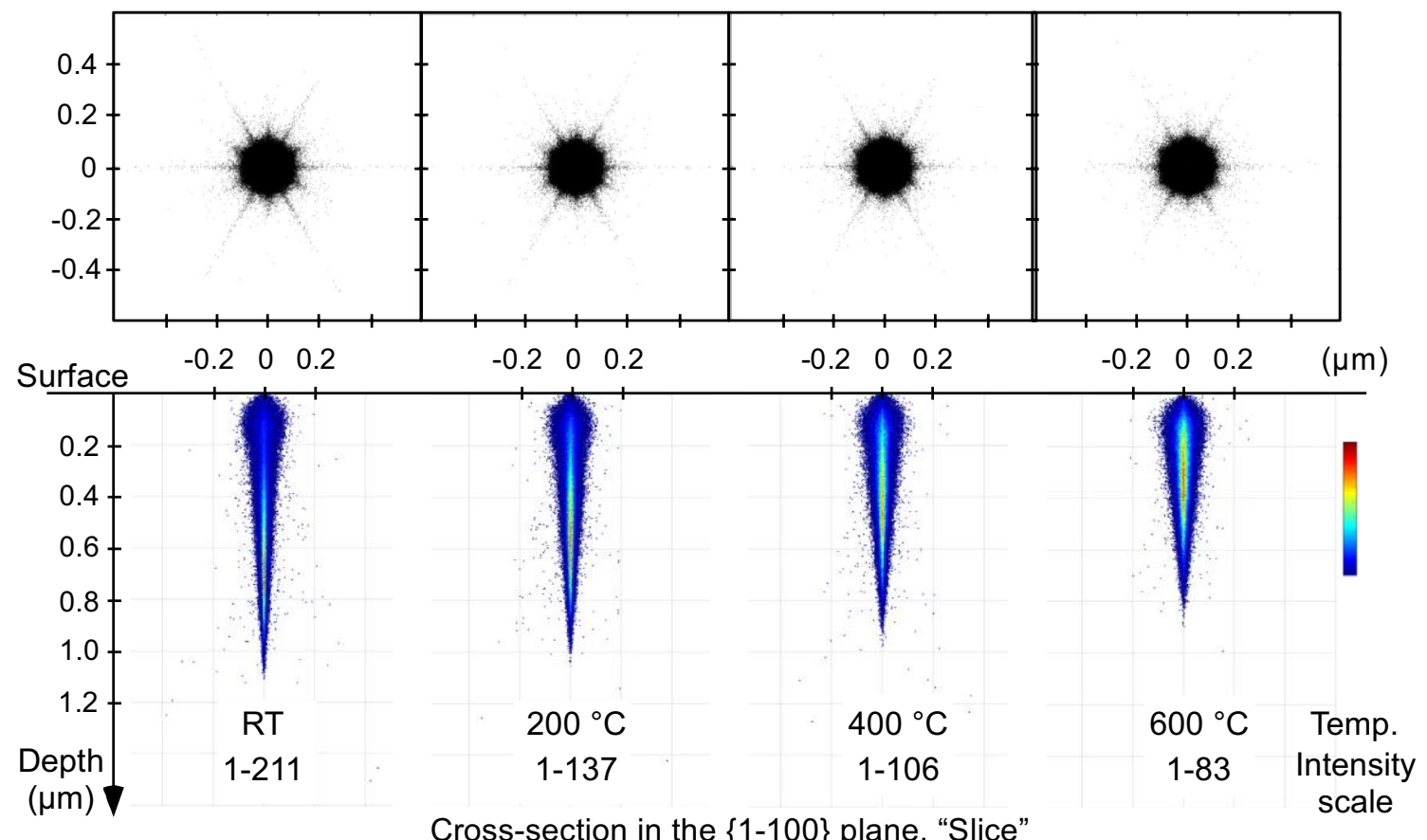

Fig. 5 3D simulation of implantation of $100 \mathrm{keV}{ }^{27} \mathrm{Al}^{+}$ions along the [000-1] direction in $4 \mathrm{H}-\mathrm{SiC}$ at RT and 200,400 and $600{ }^{\circ} \mathrm{C}$. In the simulations, an impact area of $1 \times 1 \mathrm{~nm}^{2}$ has been used. On top, a total amount of ${ }^{27} \mathrm{Al}$ ions from the whole volume shown in black and below the cross section, represented by a slice in the $\{1-100\}$ plane,

is around $0.7 \mu \mathrm{m}$ (red color). Note also that the ions are more confined to the crystal direction at RT compared to implantation performed at elevated temperatures. As the temperature increases, the channeling depth is reduced and the probability for de-channeling increases. The "cone" around the channel direction has a broader base and shorter length. The highest probability of well-channeled ions along the main track moves closer to the surface, and at $600{ }^{\circ} \mathrm{C}$, the maximum is found around $0.5 \mu \mathrm{m}$. Taking a closer look shows that there is also a small probability, a few blue dots, to find $\mathrm{Al}$ ions distributed far out laterally from the main channel. This is supported by the top view, where the six-fold symmetry of the lattice is revealed with an increased probability for the ions in the $\{1-100\}$ and $\{11-20\}$ planes. In the $\{1-100\}$ plane, the main contribution is from $<11-23>$ crystal directions, $17^{\circ}$ off from the original beam direction. In addition, a few ions are found far away from the central track.

In conclusion, if channeling implants will become a useful tool for deep box implantations in devices, at least three basic aspects have to be concerned. These are i) surface oxides, ii) implantation temperature and iii) high probability for ions to be steered into various crystal directions and planes. However, by MC calculations, the contribution of these three effects can be predicted. is displayed. The colors are used for the intensity scale. Different scales are used for different temperatures due to a higher confinement to the [000-1] channel at RT compared to implantations at elevated temperatures

\section{Summary}

The native oxide of $4 \mathrm{H}-\mathrm{SiC}$ plays a decisive role for dechanneling of aligned implantations of $\mathrm{Al}$ along the [000-1] direction. At RT, an $8 \AA$ thick amorphous layer is needed in the simulations to fit the experimental results, which is a thickness in agreement with what is expected from the native oxide. Furthermore, a decrease in thickness of the scattering non-aligned top layer from 8 to $4 \AA$ is revealed for samples heat treated at $600^{\circ} \mathrm{C}$ and sequential implanted at 200,400 and $600{ }^{\circ} \mathrm{C}$, indicating a desorption from the top layer (baking).

As the implantation temperature increases, the thermal vibrations increase and result in larger de-channeling and shallower profiles. The effect of the uppermost non-aligned layers becomes less visible after implantation at elevated temperatures. At $600{ }^{\circ} \mathrm{C}$, the contribution to de-channeling from the non-aligned top layer cannot be separated from the increasing de-channeling caused by increasing thermal vibrations of carbon and silicon atoms. According to simulations and experimental data, as the temperature is increased from RT to $600{ }^{\circ} \mathrm{C}$, the mean-square displacement is increased with $60 \%$ and, accordingly, the channeling depth is reduced. 
Acknowledgements Open access funding provided by Royal Institute of Technology. Financial support by the Swedish Research Council (VR E0510501), the Ion Technology Centre, ITC, in Sweden via VRRFI (contracts \#821-2012-5144 and \#2017-00646_9) and the Swedish Foundation for Strategic Research (SSF, contract RIF14-0053) and the Research Council of Norway through the research project FUNDAMeNT (no. 251131) and the Norwegian Micro and Nano-Fabrication Facility NorFab (no. 245963) are gratefully acknowledged.

Open Access This article is distributed under the terms of the Creative Commons Attribution 4.0 International License (http://creativeco mmons.org/licenses/by/4.0/), which permits unrestricted use, distribution, and reproduction in any medium, provided you give appropriate credit to the original author(s) and the source, provide a link to the Creative Commons license, and indicate if changes were made.

\section{References}

1. T. Kimoto, K. Kawahara, H. Niwa, N. Kaji, J. Suda, International Workshop on Junction Technology (IWJT). IEEE Proc. 2014, 1 (2014)

2. A. Hallén, M.K. Linnarsson, Surf. Coat. Tech. 306, 190 (2016)

3. D.S. Gemmell, Rev. Mod. Phys. 46, 129 (1974)

4. K. Nordlund, F. Djurabekova, G. Hobler, Phys. Rev. B 94, 214109 (2016)

5. A. Vantomme, Nucl. Instr. Meth. B 371, 12 (2016)

6. J. Wong-Leung, M.S. Janson, B.G. Svensson, J. Appl. Phys. 93, $8914(2003)$

7. A. Hallén, M.K. Linnarsson, L. Vines, Mat. Sci. Forum 963, 375 (2019)

8. M.K. Linnarsson, A. Hallén, L. Vines, B.G. Svensson, Mat. Sci. Forum 963, 382 (2019)

9. US Patent Application Publication US 2015/0028350 A1, Jan. 29, 2015, Cree Inc.
10. US Patent Application Publication US 2018/0069083 A1, Mar. 8, 2018, Cree Inc.

11. C. Önneby, C.G. Pantano, J. Vac. Sci. Technol. A15, 1597 (1997)

12. J. Lindhard, Phys. Lett. 12, 126 (1964)

13. J. Lindhard, Mat. Fys. Medd. Dan. Vid. Selsk. 34, 14 (1965)

14. S.T. Picraux, J.A. Davies, L. Eriksson, N.G.E. Johansson, J.W. Mayer, Phys. Rev. 180, 873 (1969)

15. G. Hobler, Radiat. Eff. Def. Sol. 139, 21 (1996)

16. M. Posselt, B. Schmidt, T. Feudel, N. Strecker, Mater. Sci. Eng. B71, 128 (2000)

17. G. Lulli, E. Albertazzi, M. Bianconi, R. Nipoti, M. Cervera, A. Carnera, C. Celini, J. Appl. Phys. 82, 5958 (1997)

18. G. Hobler, Nucl. Instr. Meth. B96, 155 (1995)

19. E. Morvan, P. Godignon, J. Montserrat, J. Fernández, D. Flores, J. Millán, J.P. Chante, Mat. Sci. Eng. B46, 218 (1997)

20. M.S. Janson, PhD Thesis, KTH-Royal Institute of Technology 2003, ISSN0284-0545

21. M.T. Robinson, I.M. Torrens, Phys. Rev. B 9, 5008 (1974)

22. J.F. Ziegler, J.P. Biersack, Y. Litmark, The stopping and ranges of ions in solids. Pergamon Press, Oxford (1985)

23. J.P. Biersack, L. Haggmark, Nucl. Instr. Meth. 174, 257 (1980)

24. M.S. Janson, M.K. Linnarsson, A. Hallén, B.G. Svensson, J. Appl. Phys. 96, 164 (2004)

25. G.H. Kinching, R.S. Pease, Rep. Prog. Phys. 18, 1 (1955)

26. V. Heera, W. Skorupa, Mat. Res. Symp. Proc. 438, 241 (1997)

27. Nuclear Energy Agency, data bank, computer service, https:// www.oecd-nea.org/tools/abstract/detail/nea-0919/

28. A. Zywietz, K. Karch, F. Bechstedt, Phys. Rev. B 54, 1791 (1996)

Publisher's Note Springer Nature remains neutral with regard to jurisdictional claims in published maps and institutional affiliations. 